\title{
ON THE CYLINDRICAL GREEN'S FUNCTION FOR REPRESENTATION THEORY AND ITS APPLICATIONS
}

\section{Lei Qiao}

\author{
Henan University of Economics and Law, School of Mathematics and Information Science \\ Zhengzhou 450046, P. R. China; qiaocqu@huel.edu.cn
}

\begin{abstract}
It has been known for some time that the Green's function of a planar domain can be defined in terms of the exit time of Brownian motion, and this definition has been applied to the representation theory. In this paper, we extend the notion of conformal invariance of Green's function to superharmonic functions, and use this extension to construct and estimate the cylindrical Green's function for Dirichlet boundary value problem. These considerations lead to a new proof of the representation theory of superharmonic functions defined in cylinders. We also show this estimation can be used to obtain a covering property of rarefied sets at infinity. Finally, by giving an example, we show that the reverse of this property is not true.
\end{abstract}

\section{Introduction}

In the field of analysis, Green's function $\mathcal{G}(V, W)$ on regions of $\mathbf{R}^{n}(n \geq 2)$ is formally defined to be the solution of $\mathfrak{L} \mathcal{G}(V, W)=\delta(W-V)$, where $\mathfrak{L}$ is a linear differential operator. In complex analysis, where the Laplacian is the differential operator of most importance, for a given domain $D \subseteq \mathbf{C}$ and $V \in D$ the Green's function of the Laplacian is generally defined by the following.

Definition 1.1. The Green's function $\mathcal{G}_{D}(V, W)$ on a domain $D$ is a function in $W$ on $D \backslash\{V\}$ satisfying the following properties.

(I) $\mathcal{G}_{D}(V, W)$ is harmonic and positive on $D \backslash\{V\}$.

(II) $\mathcal{G}_{D}(V, W) \rightarrow 0$ as $W \rightarrow \operatorname{bd}(D)$, where $\operatorname{bd}(D)$ is the set-theoretic boundary of $D$ (note that the boundary is to be taken in the Riemann sphere $\widehat{\mathbf{C}}:=$ $\mathbf{C} \cup\{\infty\}$, so that if $D$ is unbounded then $\infty \in D)$.

(III) $\mathcal{G}_{D}(V, W)+\frac{1}{\pi} \ln |W-V|$ extends to be continuous (and therefore harmonic) at $W=V$.

In fact, as defined here, it can be shown by standard analytic techniques that $\mathcal{G}_{D}$ satisfies $\mathfrak{L} \mathcal{G}_{D}(V, W)=\delta(W-V)$. Note that the normalization here has been chosen to align with the probabilistic considerations to follow; removing the multiplicative constant $\pi^{-1}$ from (III) would result in a solution to $\mathfrak{L G}_{D}(V, W)=\delta(W-V)$. Not every domain has a Green's function as defined above, as for instance it can be shown that no such function can exist on the punctured disk $\{0<|V|<1\}$, or more generally on a domain with isolated singularities. The Green's function is of tremendous importance in analysis on $\mathbf{R}^{n}$, including complex analysis, and the question of what domains possess a Green's function has been keenly studied by analysts over the years.

https://doi.org/10.5186/aasfm.2019.4466

2010 Mathematics Subject Classification: Primary 31B10, 35J05, 35J10, 35J40, 65N38, 65N55.

Key words: Cylindrical Green's function, cylindrical Green's potential, representation theory, covering property. 
On the other hand, the term "Green's function" has entered the vocabulary of probabilists in a way that may seem initially unrelated, namely as a measure the expected number of times that a discrete process visits a point, or the expected amount of time that a continuous process spends at a point. The reason that these two different notions have garnered the same name was discovered by Miyamoto and his collaborators in [10,9], who showed that in many cases in $\mathbf{R}^{n}$ these two notions coincide, with $\mathfrak{L}$ the Laplacian and the process in question Markov chains.

The aim of the present article is to construct the cylindrical Green's function and study the behaviors at infinity of cylindrical Green's potential and cylindrical Poisson integral.

We are motivated by the recent paper of Huang (see [7]) who constructed a Green-Sch's function for the Schrödinger operator by using the stand construction of the modified minimal Green's function for the stationary Schrödinger operator in a smooth cone. We refer to [8] and $[12,13]$ as well as to references therein for more general Green's function analysis of Schrödinger operators.

The outline of our paper is as follows. In Section 2 we recall some notations and definitions. Sections 4 and 4 are devoted to the study of behavior at infinity of cylindrical Green's potential and cylindrical Poisson integral, respectively. In Sections 5 and 6 we discuss two applications of the above results. Section 5 is devoted to give an explicit representation formula of superharmonic function in cylinders. In Section 6 we characterise the geometrical properties of rarefied sets at infinity. Finally the paper ends in Section 7, where we present one explicit example and show the reverse of the covering theorem obtained in Section 6 is not true.

\section{Preliminaries}

We keep using the same notations appearing in [9]. Let $B(V, r)$ denote the open ball with center at $V$ and radius $r$ in $\mathbf{R}^{n}$, where $V=(X, y) \in \mathbf{R}^{n}$ and $r>0$. The unit sphere in $\mathbf{R}^{n}$ is denoted by $\mathbf{S}^{n-1}$. The surface area $2 \pi^{n / 2}\{\Gamma(n / 2)\}^{-1}$ of it is denoted by $w_{n}$. For positive functions $h_{1}$ and $h_{2}$, we say that $h_{1} \lesssim h_{2}$ if $h_{1} \leq c h_{2}$ for some positive constant $c$. If $h_{1} \lesssim h_{2}$ and $h_{2} \lesssim h_{1}$, we say that $h_{1} \approx h_{2}$.

We consider the a bounded domain $\Gamma$ in $\mathbf{R}^{n-1}$ with smooth boundary $\partial \Gamma$, and study the following Dirichlet problem (see [17, p. 41])

$$
\begin{aligned}
\left(\Delta_{n-1}+\lambda\right) \phi(X) & =0 \text { in } \Gamma, \\
\phi(X) & =0 \text { on } \partial \Gamma .
\end{aligned}
$$

In the Euclidean case the above boundary value problem has the least positive eigenvalue, which is denoted by $\lambda$. The normalized eigenfunction corresponding to it is denoted by $\phi(X)$.

Let $\mathbb{H}(\Gamma)$ be the set (see $[20,21])$

$$
\mathbb{H}(\Gamma)=\Gamma \times \mathbf{R},
$$

which is called a cylinder. Let $I \subset \mathbf{R}$. The sets $\Gamma \times I$ and $\partial \Gamma \times I$ are denoted by $\mathrm{H}(\Gamma ; I)$ and $\partial \mathbb{H}(\Gamma ; I)$, respectively. We will always use the notation $\eta(V)$ to denote the distance between a point $V=(X, y) \in \mathbb{H}(\Gamma)$ and $\partial \mathbb{H}(\Gamma)$, it is well known that (see [2])

$$
\eta(V) \approx \phi(X)
$$


Definition 2.1. Let $E \subset \mathbb{H}(\Gamma)$. If there exists a sequence of open balls $\left\{B_{j}\left(V_{j}\right.\right.$, $\left.\left.r_{j}\right)\right\}_{j=1}^{\infty}\left(V_{j}=\left(X_{j}, y_{j}\right) \in \mathbb{H}(\Gamma)\right)$ such that $E \subset \cup_{j} B_{j}$, then we say that the set $E$ has a covering $\left\{r_{j}\right\}_{j=1}^{\infty}$.

Going forward, we will always use the notation $\mathcal{G}_{\Gamma}(V, W)$ be cylindrical Green's function of $\mathbb{H}(\Gamma)$, where $V, W \in \mathbb{H}(\Gamma)$. This leads one to the consideration of cylindrical Poisson kernel

$$
\mathcal{P} \mathcal{I}_{\Gamma}(V, W):=\frac{1}{c_{n}} \frac{\partial \mathcal{G}_{\Gamma}(V, W)}{\partial n_{W}}
$$

where $\partial / \partial n_{W}$ denotes the differentiation at $W \in \partial \mathbb{H}(\Gamma)$. Here, $c_{2}=2$ and $c_{n}=$ $(n-2) w_{n}$ when $n \geq 3$. This definitions appears in many different contexts, we refer the reader to $[3,4,10,15,20]$ and references therein. It follows from our assumption on $\Gamma$ that $\mathcal{P} \mathcal{I}_{\Gamma}(V, W)$ is continuous on $\partial \mathbb{H}(\Gamma)$ (see [5, Theorem 6.15]).

For any $V=(X, y) \in \mathbb{H}(\Gamma)$ and a positive measure $\nu$ in $\mathbb{H}(\Gamma)$, we use $\mathcal{G}_{\Gamma} \nu(V)$ to denote the cylindrical Green's potential by defining

$$
\mathcal{G}_{\Gamma} \nu(V):=\int_{\mathbb{H}(\Gamma)} \mathcal{G}_{\Gamma}(V, W) d \nu(W) \not \equiv+\infty
$$

The cylindrical Poisson integral on $\partial \mathbb{H}(\Gamma)$ is defined by $\mathcal{P} \mathcal{I}_{\Gamma} \mu(V)$

$$
\mathcal{P} \mathcal{I}_{\Gamma} \mu(V):=\int_{\partial \mathbb{H}(\Gamma)} \mathcal{P} \mathcal{I}_{\Gamma}(V, W) d \mu(W) \not \equiv+\infty
$$

for any $V=(X, y) \in \mathbb{H}(\Gamma)$, where $\mu$ is a positive measure on $\partial \mathbb{H}(\Gamma)$.

Definition 2.2. For the measure $\nu$ in (2.2), two positive measures $\sigma_{1}(\nu)$ and $\sigma_{2}(\nu)$ on $\mathbf{R}^{n}$ are defined by

$$
d \sigma_{1}(\nu)(W)= \begin{cases}e^{\sqrt{\lambda} y^{\prime}} \phi\left(X^{\prime}\right) d \nu(W), & W=\left(X^{\prime}, y^{\prime}\right) \in \mathbb{H}(\Gamma ;(-\infty,-1]), \\ 0, & W=\left(X^{\prime}, y^{\prime}\right) \in \mathbf{R}^{n}-\mathbb{H}(\Gamma ;(-\infty,-1]),\end{cases}
$$

and

$$
d \sigma_{2}(\nu)(W)= \begin{cases}e^{-\sqrt{\lambda} y^{\prime}} \phi\left(X^{\prime}\right) d \nu(W), & W=\left(X^{\prime}, y^{\prime}\right) \in \mathbb{H}(\Gamma ;[1,+\infty)), \\ 0, & W=\left(X^{\prime}, y^{\prime}\right) \in \mathbf{R}^{n}-\mathbb{H}(\Gamma ;[1,+\infty)),\end{cases}
$$

respectively.

Definition 2.3. For the measure $\mu$ in (2.3), two positive measures $\sigma_{1}(\mu)$ and $\sigma_{2}(\mu)$ on $\mathbf{R}^{n}$ are defined by

$$
d \sigma_{1}(\mu)(W)= \begin{cases}e^{\sqrt{\lambda} y^{\prime}} \frac{\partial \phi\left(X^{\prime}\right)}{\partial n_{X^{\prime}}} d \mu(W), & W=\left(X^{\prime}, y^{\prime}\right) \in \partial \mathbb{H}(\Gamma ;(-\infty,-1]), \\ 0, & W=\left(X^{\prime}, y^{\prime}\right) \in \mathbf{R}^{n}-\partial \mathbf{H}(\Gamma ;(-\infty,-1])\end{cases}
$$

and

$$
d \sigma_{2}(\mu)(W)= \begin{cases}e^{-\sqrt{\lambda} y^{\prime}} \frac{\partial \phi\left(X^{\prime}\right)}{\partial n_{X^{\prime}}} d \mu(W), & W=\left(X^{\prime}, y^{\prime}\right) \in \partial \mathbb{H}(\Gamma ;[1,+\infty)) \\ 0, & W=\left(X^{\prime}, y^{\prime}\right) \in \mathbf{R}^{n}-\partial \mathbb{H}(\Gamma ;[1,+\infty)),\end{cases}
$$

respectively. 
Definition 2.4. For two measures $\mu$ and $\nu$ in (2.3) and (2.2), respectively, two positive measures $\sigma_{1}(\mu, \nu)$ and $\sigma_{2}(\mu, \nu)$ on $\mathbf{R}^{n}$ are defined by

$$
d \sigma_{1}(\mu, \nu)(W)=\left\{\begin{array}{lc}
e^{\sqrt{\lambda} y^{\prime}} d \sigma(\mu, \nu)(W), & W=\left(X^{\prime}, y^{\prime}\right) \in \mathbb{H}(\Gamma ;(-\infty,-1]) \\
& \cup \partial \mathbb{H}(\Gamma ;(-\infty,-1]), \\
0, & W=\left(X^{\prime}, y^{\prime}\right) \in \mathbf{R}^{n}-\mathbb{H}(\Gamma ;(-\infty,-1]) \\
& -\partial \mathbb{H}(\Gamma ;(-\infty,-1])
\end{array}\right.
$$

and

$$
d \sigma_{2}(\mu, \nu)(W)=\left\{\begin{array}{lc}
e^{-\sqrt{\lambda} y^{\prime}} d \sigma(\mu, \nu)(W), & W=\left(X^{\prime}, y^{\prime}\right) \in \mathbb{H}(\Gamma ;[1,+\infty)) \\
& \cup \partial \mathbb{H}(\Gamma ;[1,+\infty)) \\
0, & \left(X^{\prime}, y^{\prime}\right) \in \mathbf{R}^{n}-\mathbb{H}(\Gamma ;[1,+\infty)) \\
& -\partial \mathbb{H}(\Gamma ;[1,+\infty))
\end{array}\right.
$$

respectively, where

$d \sigma(\mu, \nu)(W)= \begin{cases}\phi\left(X^{\prime}\right) d \nu(W), & W=\left(X^{\prime}, y^{\prime}\right) \in \mathbb{H}(\Gamma ;(-\infty,-1]) \cup \mathbb{H}(\Gamma ;[1,+\infty)), \\ \frac{\partial \phi\left(X^{\prime}\right)}{\partial n_{X^{\prime}}} d \mu(W), & W=\left(X^{\prime}, y^{\prime}\right) \in \partial \mathbb{H}(\Gamma ;(-\infty,-1]) \cup \partial \mathbb{H}(\Gamma ;[1,+\infty)) .\end{cases}$

Remark 2.1. It should be noticed that the total masses $\sigma_{1}(\nu)$ are $\sigma_{1}(\mu)$ are finite from Lemmas 3.2 and 4.2, respectively. The proofs of them will be given later in Sections 3 and 4 , respectively. So, it is easy to see that $\sigma_{1}(\mu, \nu)$ is also finite. Similarly, the total masses of $\sigma_{2}(\nu), \sigma_{2}(\mu)$ and $\sigma_{2}(\mu, \nu)$ are also finite.

Definition 2.5. Let $\gamma>0$ and $\varrho$ be any positive measure on $\mathbf{R}^{n}$ satisfying $\|\varrho\|<\infty$. For each point $V \in \mathbb{H}(\Gamma)$, we define the maximal function $\mathcal{M}(V ; \varrho, \gamma)$ by

$$
\mathcal{M}(V ; \varrho, \gamma):=\sup _{0<\rho \leq \frac{\eta(V)}{2}} \frac{\varrho(B(V, \rho))}{\rho^{\gamma}} .
$$

This leads one to the following exceptional set

$$
\mathcal{E}(\epsilon ; \varrho, \gamma):=\{V \in \mathbb{H}(\Gamma) ; \mathcal{M}(V ; \varrho, \gamma)>\epsilon\},
$$

where $\epsilon$ is a sufficiently small positive number.

Remark 2.2. Let $V \in \mathbb{H}(\Gamma)$ satisfy $\varrho(\{V\})>0$. Then $\mathcal{M}(V ; \varrho, \gamma)=+\infty$. It follows that

$$
\mathcal{E}(\epsilon ; \varrho, \gamma) \supset\{V \in \mathbb{H}(\Gamma) ; \varrho(\{V\})>0\}
$$

\section{Behaviors at infinity of the cylindrical Green's potential}

In this section we show how calculating the asymptotic behaviors at infinity of cylindrical Green's potential can yield two inequalities. Let us first introduce the following estimates of cylindrical Green's function, which are fundamental (see $[20,21])$.

Lemma 3.1. Let $V=(X, y) \in \mathbb{H}(\Gamma)$ and $W=\left(X^{\prime}, y^{\prime}\right) \in \mathbb{H}(\Gamma)$ such that $y^{\prime}>y+1$. Then

$$
\mathcal{G}_{\Gamma}(V, W) \approx e^{\sqrt{\lambda} y} e^{-\sqrt{\lambda} y^{\prime}} \phi(X) \phi\left(X^{\prime}\right) .
$$

Let $V=(X, y) \in \mathbb{H}(\Gamma)$ and $W=\left(X^{\prime}, y^{\prime}\right) \in \mathbb{H}(\Gamma)$ such that $y^{\prime}<y-1$. Then

$$
\mathcal{G}_{\Gamma}(V, W) \approx e^{-\sqrt{\lambda} y} e^{\sqrt{\lambda} y^{\prime}} \phi(X) \phi\left(X^{\prime}\right) .
$$


As was alluded to earlier, the following proposition and its proof must certainly be known (see [9, Lemma 1]), but it seems difficult to locate a simple statement in the literature (perhaps for want of applications). For the benefit of the reader, we therefore provide a quick proof.

Lemma 3.2. Let $V_{i}=\left(X_{i}, y_{i}\right) \in \mathbb{H}(\Gamma), y_{i} \rightarrow-\infty(i \rightarrow+\infty)$ be a sequence of points such that $\mathcal{G}_{\Gamma} \nu\left(V_{i}\right)<+\infty$ for $i=1,2, \ldots$, where $\nu$ is a positive measure on $\mathrm{H}(\Gamma)$. Then we have

$$
\int_{\mathbb{H}(\Gamma ;(-\infty, l))} e^{\sqrt{\lambda} y^{\prime}} \phi\left(X^{\prime}\right) d \nu\left(X^{\prime}, y^{\prime}\right)<+\infty
$$

and

$$
\lim _{R \rightarrow-\infty} e^{2 \sqrt{\lambda} R} \int_{\mathbb{H}(\Gamma ;(R,+\infty))} e^{-\sqrt{\lambda} y^{\prime}} \phi\left(X^{\prime}\right) d \nu\left(X^{\prime}, y^{\prime}\right)=0
$$

for a real number $l$.

Proof. Let $l$ be a real number such that $V_{1}=\left(X_{1}, y_{1}\right) \in \mathbb{H}(\Gamma ;[l+1,+\infty))$. Then we have

$$
e^{-\sqrt{\lambda} y_{1}} \phi\left(X_{1}\right) \int_{\mathbb{H}(\Gamma ;(-\infty, l))} e^{\sqrt{\lambda} y^{\prime}} \phi\left(X^{\prime}\right) d \nu\left(X^{\prime}, y^{\prime}\right) \lesssim \mathcal{G}_{\Gamma} \nu\left(V_{1}\right)<+\infty
$$

from (3.2), which gives (3.3). It follows from (3.3) that

$$
\int_{\mathbb{H}(\Gamma ;(-\infty, A))} e^{\sqrt{\lambda} y^{\prime}} \phi\left(X^{\prime}\right) d \nu\left(X^{\prime}, y^{\prime}\right)<\frac{\epsilon}{2},
$$

where $A$ is a sufficiently small number.

Let $V_{i}=\left(X_{i}, y_{i}\right) \in \mathbb{H}(\Gamma ;(-\infty, A-1))$. Then we have

$$
e^{\sqrt{\lambda} y_{i}} \phi\left(X_{i}\right) \int_{\mathbb{H}(\Gamma ;(A,+\infty))} e^{-\sqrt{\lambda} y^{\prime}} \phi\left(X^{\prime}\right) d \nu\left(X^{\prime}, y^{\prime}\right) \lesssim \mathcal{G}_{\Gamma} \nu\left(V_{i}\right)<+\infty
$$

from (3.1).

Let $R<A$. Then we have

$$
\begin{aligned}
& e^{2 \sqrt{\lambda} R} \int_{\mathbb{H}(\Gamma ;(R,+\infty))} e^{-\sqrt{\lambda} y^{\prime}} \phi\left(X^{\prime}\right) d \nu\left(X^{\prime}, y^{\prime}\right) \\
& \lesssim e^{2 \sqrt{\lambda} R} \int_{\mathbb{H}(\Gamma ;(R, A))} e^{-\sqrt{\lambda} y^{\prime}} \phi\left(X^{\prime}\right) d \nu\left(X^{\prime}, y^{\prime}\right)+e^{2 \sqrt{\lambda} R} \int_{\mathbb{H}(\Gamma ;(A,+\infty))} e^{-\sqrt{\lambda} y^{\prime}} \phi\left(X^{\prime}\right) d \nu\left(X^{\prime}, y^{\prime}\right) \\
& \lesssim \int_{\mathbb{H}(\Gamma ;(R, A))} e^{\sqrt{\lambda} y^{\prime}} \phi\left(X^{\prime}\right) d \nu\left(X^{\prime}, y^{\prime}\right)+e^{2 \sqrt{\lambda} R} \int_{\mathbb{H}(\Gamma ;(A,+\infty))} e^{-\sqrt{\lambda} y^{\prime}} \phi\left(X^{\prime}\right) d \nu\left(X^{\prime}, y^{\prime}\right) \lesssim \epsilon
\end{aligned}
$$

from (3.5) and (3.6), which gives (3.4).

Lemma 3.3. Let $\gamma$ and $\varrho$ be as in Definition 2.5. Then the exceptional set $\mathcal{E}(\epsilon ; \varrho, \gamma)$ has a covering $\left\{r_{j}\right\}_{j=1}^{\infty}$ satisfying

$$
\sum_{j=1}^{\infty}\left(r_{j}\right)^{\gamma}<+\infty
$$

Proof. Let $j$ be a positive integer such that $j \geq 2$. Then we define

$$
\mathcal{E}_{j}(\epsilon ; \varrho, \gamma)=\mathcal{E}(\epsilon ; \varrho, \gamma) \cap I_{j}
$$

where

$$
I_{j}=\{V=(X, y) \in \mathbb{H}(\Gamma) ; j \leq|y|<j+1\}
$$


Let $V=(X, y) \in \mathcal{E}_{j}(\epsilon ; \varrho, \gamma)$. Then

$$
\{\rho(V)\}^{\gamma} \leq \epsilon^{-1} \varrho(B(V, \rho(V))) \leq \epsilon^{-1}\|\varrho\|,
$$

where $\rho(V)$ is a positive number satisfying $0<\rho(V) \leq \frac{\eta(V)}{2}$.

Since $\mathcal{E}_{j}(\epsilon ; \varrho, \gamma)$ has a trivial covering $\left\{B(V, \rho(V)) ; V^{2} \in \mathcal{E}_{j}(\epsilon ; \varrho, \gamma)\right\}$ such that

$$
\sup _{V \in \mathcal{E}_{j}(\epsilon ; \varrho, \gamma)} \rho(V) \leq \epsilon^{-\frac{1}{\gamma}}\|\varrho\|^{\frac{1}{\gamma}}<+\infty
$$

by the Besicovitch covering theorem there exists a countable subfamily $\left\{B\left(V_{j, i}\right.\right.$, $\left.\left.\rho_{j, i}\right)\right\}\left(\rho_{j, i}=\rho\left(V_{j, i}\right)\right)$ (see [11, Theorem 10.3]), which covers $\mathcal{E}_{j}(\epsilon ; \varrho, \gamma)$ and intersects each other at most $N$ times, where $N$ depends only on the dimension $n$.

It follows that (3.7)

$$
\epsilon \sum_{i}\left(\rho_{j, i}\right)^{\gamma} \leq \sum_{i} \varrho\left(B\left(V_{j, i}, \rho_{j, i}\right)\right) \leq N \varrho\left(I_{j-1} \cup I_{j} \cup I_{j+1}\right)
$$

from $B(V, \rho(V)) \cap I_{j+2}=\emptyset$ and $B(V, \rho(V)) \cap I_{j-2}=\emptyset$ for any $V \in \mathcal{E}_{j}(\epsilon ; \varrho, \gamma)$.

So we know that $\cup_{j} \mathcal{E}_{j}(\epsilon ; \varrho, \gamma)$ is covered by a sequence of balls $\left\{B\left(V_{j, i}, \rho_{j, i}\right)\right\}(j=$ $2,3,4, \ldots ; i=1,2,3, \ldots)$ such that

$$
\sum_{j, i}\left(\rho_{j, i}\right)^{\gamma} \leq 3 N\|\varrho\| \epsilon^{-1}
$$

Since

$$
\bigcup_{j=2}^{\infty} \mathcal{E}_{j}(\epsilon ; \varrho, \gamma)=\{V=(X, y) \in \mathbb{H}(\Gamma) ;|y| \geq 2\} \cap \mathcal{E}(\epsilon ; \varrho, \gamma),
$$

it follows that $\mathcal{E}(\epsilon ; \varrho, \gamma)$ is covered by a sequence of balls $\left\{B\left(V_{j, i}, \rho_{j, i}\right), B\left(V_{1}, 3\right)\right\}(j=$ $2,3,4, \ldots ; i=1,2,3, \ldots)$ such that

$$
\sum_{j, i}\left(\rho_{j, i}\right)^{\gamma} \leq 3 N\|\varrho\| \epsilon^{-1}+3^{\gamma}<+\infty
$$

where $B\left(V_{1}, 3\right)\left(V_{1}=(X, 0) \in \mathbf{R}^{n}\right)$ is the ball which covers the set $\mathbb{H}(\Gamma ;(-2,2))$.

In 2014, Wang (see [18]) presented the existence and uniqueness of solutions of the Neumann problems with bounded harmonic for the boundary value problems in a half plane. Similar results for solutions of the Dirichlet problems with a rapidly growing functions in certain unbounded domains, we refer readers to the papers by Ramachandran (see [16]) and Ren \& Yang (see [19]). Motivated by the above results, our first aim is to obtain boundary behaviors of cylindrical Green's potential.

Theorem 3.1. Let $\nu$ be as in (2.2) and $\gamma>n-2$. Then the subset $\mathcal{E}\left(\epsilon ; \sigma_{1}(\nu), \gamma\right)$ (resp. $\left.\mathcal{E}\left(\epsilon ; \sigma_{2}(\nu), \gamma\right)\right)$ of $\mathbb{H}(\Gamma)$ has a covering $\left\{r_{j}\right\}_{j=0}^{\infty}$ satisfying

$$
\sum_{j=0}^{\infty}\left(r_{j}\right)^{\gamma}<+\infty
$$

such that

$$
\begin{gathered}
\lim _{y \rightarrow-\infty, V=(X, y) \in \mathbb{H}(\Gamma)-\mathcal{E}\left(\epsilon ; \sigma_{1}(\nu), \gamma\right)} e^{\sqrt{\lambda} y} \phi^{n-\gamma-1}(X) \mathcal{G}_{\Gamma} \nu(V)=0 . \\
\left(\text { resp. } \lim _{y \rightarrow+\infty, V=(X, y) \in \mathbb{H}(\Gamma)-\mathcal{E}\left(\epsilon ; \sigma_{2}(\nu), \gamma\right)} e^{-\sqrt{\lambda} y} \phi^{n-\gamma-1}(X) \mathcal{G}_{\Gamma} \nu(V)=0 .\right)
\end{gathered}
$$


Proof. Let $R$ be a sufficiently small number such that $R \geq y+1$ and $V=(X, y) \in$ $\mathbb{H}(\Gamma ;(-\infty, R))-\mathcal{E}\left(\epsilon ; \sigma_{1}(\nu), \gamma\right)$. If we can show that

$$
\mathcal{G}_{\Gamma} \nu(V) \lesssim e^{-\sqrt{\lambda} y} \phi^{\gamma-n+1}(X) \epsilon,
$$

then we obtain that (3.9) holds.

We write

$$
\mathcal{G}_{\Gamma} \nu(V)=\mathcal{U}_{1}(V)+\mathcal{U}_{2}(V)+\mathcal{U}_{3}(V)
$$

where

$$
\begin{aligned}
& \mathcal{U}_{1}(V)=\int_{\mathbb{H}(\Gamma ;(-\infty, y-1))} \mathcal{G}_{\Gamma}(V, W) d \nu(W), \\
& \mathcal{U}_{2}(V)=\int_{\mathbb{H}(\Gamma ;[y-1, y+1])} \mathcal{G}_{\Gamma}(V, W) d \nu(W)
\end{aligned}
$$

and

$$
\mathcal{U}_{3}(V)=\int_{\mathbb{H}(\Gamma ;(y+1,+\infty))} \mathcal{G}_{\Gamma}(V, W) d \nu(W)
$$

We obtain the following estimates

$$
\mathcal{U}_{1}(V) \lesssim e^{-\sqrt{\lambda} y} \phi(X) \int_{\mathbb{H}(\Gamma ;(-\infty, y-1))} e^{\sqrt{\lambda} y^{\prime}} \phi\left(X^{\prime}\right) d \nu\left(X^{\prime}, y^{\prime}\right) \lesssim e^{-\sqrt{\lambda} y} \phi(X) \epsilon
$$

and

$$
\mathcal{U}_{3}(V) \lesssim e^{\sqrt{\lambda} y} \phi(X) \int_{\mathbb{H}(\Gamma ;(y+1,+\infty))} e^{-\sqrt{\lambda} y^{\prime}} \phi\left(X^{\prime}\right) d \nu\left(X^{\prime}, y^{\prime}\right) \lesssim e^{-\sqrt{\lambda} y} \phi(X) \epsilon
$$

from Lemmas 3.1 and 3.2.

Since

$$
\mathcal{G}_{\Gamma}(V, W) \lesssim|V-W|^{2-n}
$$

for any $W \in \mathbb{H}(\Gamma)$, we have

$$
\mathcal{U}_{2}(V) \lesssim e^{-\sqrt{\lambda} y} \int_{\mathbb{H}(\Gamma ;[y-1, y+1])} \eta^{-1}(W)|V-W|^{2-n} d \sigma_{1}(\nu)(W)
$$

Now put

$$
H_{i}(V)=\left\{W \in \mathbb{H}(\Gamma ;[y-1, y+1]) ; \eta(V) \leq 2^{i+1}|V-W|<2 \eta(V)\right\},
$$

where $i=1,2,3, \ldots$. For $V \notin \mathcal{E}\left(\epsilon ; \sigma_{1}(\nu), \gamma\right)$, it follows that

$$
\mathcal{U}_{2}(V) \lesssim e^{-\sqrt{\lambda} y} \sum_{i=1}^{\infty} \int_{H_{i}(V)} \frac{d \sigma_{1}(\nu)(W)}{|V-W|^{n-2} \eta(W)}
$$

from Remark 2.2.

It is easy to see that $2 \eta(W) \geq \eta(V)$ for any $W \in H_{i}(V)(i=1,2,3, \ldots)$, which together with (2.1), gives that

$$
\begin{aligned}
\int_{H_{i}(V)} \frac{d \sigma_{1}(\nu)(W)}{|V-W|^{n-2} \eta(W)} & \lesssim 2^{(i+1)(n-2)} \sigma_{1}(\nu)\left(H_{i}(V)\right) \eta^{1-n}(V) \\
& \lesssim 2^{(i+1)(n-2)} \sigma_{1}(\nu)\left(B\left(V, 2^{-i} \eta(V)\right)\right) \eta^{1-n}(V) \\
& \lesssim \mathcal{M}\left(V ; \sigma_{1}(\nu), \gamma\right) \phi^{\gamma-n+1}(X) 2^{i(n-\gamma-2)} .
\end{aligned}
$$


It follows that

$$
\mathcal{U}_{2}(V) \lesssim e^{-\sqrt{\lambda} y} \phi^{\gamma-n+1}(X) \epsilon \sum_{i=1}^{\infty} 2^{i(n-\gamma-2)} \lesssim e^{-\sqrt{\lambda} y} \phi^{\gamma-n+1}(X) \epsilon
$$

because $V=(X, y) \notin \mathcal{E}\left(\epsilon ; \sigma_{1}(\nu), \gamma\right)$.

Combing (3.12), (3.13), (3.14) and (3.16), we know that (3.11) holds. It is easy to see that there exists an additional finite ball $B_{0}$ covering $C_{n}(\Gamma ;[R, 0])$. So $(3.8)$ holds from Lemma 3.3 and Remark 2.1. Similarly, we prove that (3.10) holds. These complete the proof.

Corollary 3.1. If the condition (2.2) is replaced by a weaker condition

$$
\int_{\mathbb{H}(\Gamma)} e^{-\sqrt{\lambda}\left|y^{\prime}\right|} \phi\left(X^{\prime}\right) d \nu\left(X^{\prime}, y^{\prime}\right)<\infty .
$$

Then the conclusions of Theorem 3.1 still hold true.

\section{Behaviors at infinity of the cylindrical Poisson integral}

The present section is devoted to the study of limiting behavior of the cylindrical Poisson integral near infinity. We first state a simple lemma which follows from Lemma 3.1.

Lemma 4.1. Let $V=(X, y) \in \mathbb{H}(\Gamma)$ and $W=\left(X^{\prime}, y^{\prime}\right) \in \partial \mathbb{H}(\Gamma)$ such that $y^{\prime}>y+1$. Then we have

$$
\mathcal{P} \mathcal{I}_{\Gamma}(V, W) \approx e^{\sqrt{\lambda} y} e^{-\sqrt{\lambda} y^{\prime}} \phi(X) \frac{\partial \phi\left(X^{\prime}\right)}{\partial n_{X^{\prime}}} .
$$

Let $V=(X, y) \in \mathbb{H}(\Gamma)$ and $W=\left(X^{\prime}, y^{\prime}\right) \in \partial \mathbb{H}(\Gamma)$ such that $y^{\prime}<y-1$. Then we have

$$
\mathcal{P} \mathcal{I}_{\Gamma}(V, W) \approx e^{-\sqrt{\lambda} y} e^{\sqrt{\lambda} y^{\prime}} \phi(X) \frac{\partial \phi\left(X^{\prime}\right)}{\partial n_{X^{\prime}}} .
$$

Let $V=(X, y) \in \mathbb{H}(\Gamma)$ and $W=\left(X^{\prime}, y^{\prime}\right) \in \partial \mathbb{H}(\Gamma)$ such that $y-1 \leq y^{\prime} \leq y+1$. Then we have (see [1, p. 124])

$$
\mathcal{P} \mathcal{I}_{\Gamma}(V, W) \lesssim \frac{\phi(X)}{|V-W|^{n}} \frac{\partial \phi\left(X^{\prime}\right)}{\partial n_{X^{\prime}}} .
$$

Lemma 4.2. (see $\left[9\right.$, Lemma 4]) Let $V_{i}=\left(X_{i}, y_{i}\right) \in \mathbb{H}(\Gamma), y_{i} \rightarrow-\infty(i \rightarrow+\infty)$ be a sequence of points such that $\mathcal{P} \mathcal{I}_{\Gamma} \mu\left(V_{i}\right)<+\infty(i=1,2, \ldots)$, where $\mu$ is a positive measure on $\partial \mathrm{H}(\Gamma)$. Then we have

$$
\int_{\partial \mathbb{H}(\Gamma ;(-\infty, l))} e^{\sqrt{\lambda} y^{\prime}} \frac{\partial \phi\left(X^{\prime}\right)}{\partial n_{X^{\prime}}} d \mu\left(X^{\prime}, y^{\prime}\right)<+\infty
$$

and

$$
\lim _{R \rightarrow-\infty} e^{2 \sqrt{\lambda} R} \int_{\partial \mathbb{H}(\Gamma ;(R,+\infty))} e^{-\sqrt{\lambda} y^{\prime}} \frac{\partial \phi\left(X^{\prime}\right)}{\partial n_{X^{\prime}}} d \mu\left(X^{\prime}, y^{\prime}\right)=0
$$

for a real number $l$.

Proof. We can prove above result by the same method as we did in the proof of Lemma 3.2 and using Lemma 4.1 in place of Lemma 3.1. 
Lemma 4.3. (see [1]) Let $\mathcal{G}_{\mathbb{H}\left(\Gamma ;\left(y_{1}, y_{2}\right)\right)}(\cdot, \cdot)$ be cylindrical Green's function in the truncated cylinder $\mathbb{H}\left(\Gamma ;\left(y_{1}, y_{2}\right)\right)$, where $-\infty<y_{1}<y_{2}<\infty$. Then we have

$$
\frac{\partial \mathcal{G}_{\mathbb{H}\left(\Gamma ;\left(y_{1}, y_{2}\right)\right)}\left(V_{0},\left(X_{2}, y_{2}\right)\right)}{\partial y} \approx-\phi\left(X_{0}\right) \phi\left(X_{2}\right)
$$

and

$$
\frac{\partial \mathcal{G}_{\mathrm{H}\left(\Gamma ;\left(y_{1}, y_{2}\right)\right)}\left(V_{0},\left(X_{1}, y_{1}\right)\right)}{\partial y} \approx \phi\left(X_{0}\right) \phi\left(X_{1}\right)
$$

for any $V_{0}=\left(X_{0}, y_{0}\right) \in \mathbb{H}\left(\Gamma ;\left(y_{1}, y_{2}\right)\right)$.

With the help of above lemmas, next we state the main theorem of the present section. Similar results for asymptotic behaviors of conical Poisson integrals and their applications, we refer readers to the papers by Qiao (see $[15,14]$ ).

Theorem 4.1. Let $\mu$ be as in (2.3) and $\gamma \geq 0$. Then the subset $\mathcal{E}\left(\epsilon ; \sigma_{1}(\mu), \gamma\right)$ (resp. $\left.\mathcal{E}\left(\epsilon ; \sigma_{2}(\mu), \gamma\right)\right)$ of $\mathbb{H}(\Gamma)$ has a covering $\left\{r_{j}\right\}_{j=0}^{\infty}$ satisfying (3.8) such that

$$
\begin{gathered}
\lim _{y \rightarrow-\infty, V=(X, y) \in \mathbb{H}(\Gamma)-\mathcal{E}\left(\epsilon ; \sigma_{1}(\mu), \gamma\right)} e^{\sqrt{\lambda} y} \phi^{n-\gamma-1}(X) \mathcal{P} \mathcal{I}_{\Gamma} \mu(V)=0 . \\
\text { (resp. } \left.\lim _{y \rightarrow+\infty, V=(X, y) \in \mathbb{H}(\Gamma)-\mathcal{E}\left(\epsilon ; \sigma_{2}(\mu), \gamma\right)} e^{-\sqrt{\lambda} y} \phi^{n-\gamma-1}(X) \mathcal{P} \mathcal{I}_{\Gamma} \mu(V)=0 .\right)
\end{gathered}
$$

Proof. We only prove (4.2). The proof of (4.3) is similar. Let $R$ is a sufficiently small number such that $R \geq y+1$. For any point $V=(X, y) \in \mathbb{H}(\Gamma ;(-\infty, R))-$ $\mathcal{E}\left(\epsilon ; \sigma_{1}(\mu), \gamma\right)$, we write

$$
\mathcal{P} \mathcal{I}_{\Gamma} \mu(V)=\mathcal{U}_{4}(V)+\mathcal{U}_{5}(V)+\mathcal{U}_{6}(V)
$$

where

and

$$
\begin{aligned}
& \mathcal{U}_{4}(V)=\int_{\partial \mathrm{H}(\Gamma ;(-\infty, y-1))} \mathcal{P} \mathcal{I}_{\Gamma}(V, W) d \mu(W), \\
& \mathcal{U}_{5}(V)=\int_{\partial \mathrm{H}(\Gamma ;[y-1, y+1])} \mathcal{P} \mathcal{I}_{\Gamma}(V, W) d \mu(W)
\end{aligned}
$$

$$
\mathcal{U}_{6}(V)=\int_{\partial \mathrm{H}(\Gamma ;(y+1,+\infty))} \mathcal{P} \mathcal{I}_{\Gamma}(V, W) d \mu(W) .
$$

We have the following estimates

$$
\mathcal{U}_{4}(V) \lesssim e^{-\sqrt{\lambda} y} \phi(X) \int_{\partial \mathrm{H}(\Gamma ;(-\infty, y-1))} e^{\sqrt{\lambda} y^{\prime}} \frac{\partial \phi\left(X^{\prime}\right)}{\partial n_{X^{\prime}}} d \mu\left(X^{\prime}, y^{\prime}\right) \lesssim e^{-\sqrt{\lambda} y} \phi(X) \epsilon
$$

and

$$
\mathcal{U}_{6}(V) \lesssim e^{\sqrt{\lambda} y} \phi(X) \int_{\partial \mathrm{H}(\Gamma ;(y+1,+\infty))} e^{-\sqrt{\lambda} y^{\prime}} \frac{\partial \phi\left(X^{\prime}\right)}{\partial n_{X^{\prime}}} d \mu\left(X^{\prime}, y^{\prime}\right) \lesssim e^{-\sqrt{\lambda} y} \phi(X) \epsilon
$$

from Lemmas 4.1 and 4.2. To estimate $\mathcal{U}_{5}(V)$. For any $V=(X, y) \in \Lambda(k)$, where $k$ is a sufficiently small positive number and

$$
\Lambda(k)=\left\{V=(X, y) \in \mathbb{H}(\Gamma ;(-\infty, 0)) ; \inf _{(X, z) \in \partial \mathbb{H}(\Gamma)}|(X, y)-(X, z)|<k\right\} .
$$

Then we have

$$
\partial \mathrm{H}(\Gamma ;[y-1, y+1]) \subset B\left(V, \frac{\eta(V)}{2}\right) .
$$

And we divide $\mathbb{H}(\Gamma)$ into two sets $\Lambda(k)$ and $\mathbb{H}(\Gamma)-\Lambda(k)$. 
It is easy to see that $|V-W| \geq k^{\prime}$ for any $V=(X, y) \in \mathbb{H}(\Gamma)-\Lambda(k)$ and any $W \in \partial \mathrm{H}(\Gamma)$, where $k^{\prime}$ is a positive number. It follow from (4.1) and Lemma 4.1 that

$$
\mathcal{U}_{5}(V) \lesssim e^{-\sqrt{\lambda} y} \phi(X) \epsilon
$$

Now we consider the case $V \in \Lambda(k)$. Define

$$
I_{i}(V)=\left\{W \in \partial \mathbb{H}(\Gamma ;[y-1, y+1]) ; 2^{i-1} \eta(V) \leq|V-W|<2^{i} \eta(V)\right\},
$$

$i=0,1,2,3, \ldots$ Let $i(V)$ be a positive integer satisfy $I_{i}(V)=\emptyset$ for $i \geq i(V)+1$.

Then it follows that

$$
\mathcal{U}_{5}(V) \lesssim \sum_{i=1}^{i(V)} \int_{I_{i}(V)} e^{-\sqrt{\lambda} y^{\prime}}|V-W|^{-n} \phi\left(X^{\prime}\right) d \sigma_{1}(\mu)(W)
$$

from $\left\{W \in \mathbf{R}^{n}:|V-W|<\eta(V)\right\} \cap \partial \mathbb{H}(\Gamma)=\varnothing$.

We have

$$
\int_{I_{i}(V)} e^{-\sqrt{\lambda} y^{\prime}}|V-W|^{-n} \phi\left(X^{\prime}\right) d \sigma_{1}(\mu)(W) \lesssim e^{-\sqrt{\lambda} y} \phi^{\gamma-n+1}(X) \frac{\sigma_{1}(\mu)\left(I_{i}(V)\right)}{\left(2^{i} \eta(V)\right)^{\gamma}}
$$

for $i=0,1,2, \ldots, i(V)$ from (2.1) It follows that

$$
\frac{\sigma_{1}(\mu)\left(I_{i}(V)\right)}{\left(2^{i} \eta(V)\right)^{\gamma}} \lesssim \frac{\sigma_{1}(\mu)\left(B\left(V, 2^{i} \eta(V)\right)\right)}{\left(2^{i} \eta(V)\right)^{\gamma}} \leq \mathcal{M}\left(V ; \sigma_{1}(\mu), \gamma\right) \lesssim \epsilon
$$

for $i=0,1,2, \ldots, i(V)-1$ and

$$
\frac{\sigma_{1}(\mu)\left(I_{i(V)}(V)\right)}{\left(2^{i(V)} \eta(V)\right)^{\gamma}} \lesssim \frac{\sigma_{1}(\mu)\left(B\left(V, 2^{-1} \eta(V)\right)\right)}{\left(2^{-1} \eta(V)\right)^{\gamma}} \lesssim \epsilon,
$$

where $V=(X, y) \notin \mathcal{E}\left(\epsilon ; \sigma_{1}(\mu), \gamma\right)$. So

$$
\mathcal{U}_{5}(V) \lesssim e^{-\sqrt{\lambda} y} \phi^{\gamma-n+1}(X) \epsilon .
$$

For any $V=(X, y) \in \mathbb{H}(\Gamma ;(-\infty, R))-\mathcal{E}\left(\epsilon ; \sigma_{1}(\mu), \gamma\right)$, combining (4.4), (4.5), (4.6) and (4.7), we have

$$
\mathcal{P} \mathcal{I}_{\Gamma} \mu(V) \lesssim e^{-\sqrt{\lambda} y} \phi^{\gamma-n+1}(X) \epsilon .
$$

It is easy to see that there exists an additional finite ball $B_{0}$ covering $C_{n}(\Gamma ;[R, 0])$. So it follows that (3.8) holds from Lemma 3.3 and Remark 2.1. These complete the proof.

Corollary 4.1. Let $\mu$ be as in (2.3). Then we have

$$
\begin{gathered}
\lim _{y \rightarrow-\infty, V=(X, y) \in \mathbb{H}(\Gamma)} e^{\sqrt{\lambda} y} \phi^{n-1}(X) \mathcal{P} \mathcal{I}_{\Gamma} \mu(V)=0 . \\
\text { (resp. } \left.\lim _{y \rightarrow+\infty, V=(X, y) \in \mathbb{H}(\Gamma)} e^{-\sqrt{\lambda} y} \phi^{n-1}(X) \mathcal{P} \mathcal{I}_{\Gamma} \mu(V)=0 .\right)
\end{gathered}
$$

Corollary 4.2. If the condition (2.3) is replaced by

$$
\int_{\partial \mathbb{H}(\Gamma)} e^{-\sqrt{\lambda}\left|y^{\prime}\right|} \frac{\partial \phi\left(X^{\prime}\right)}{\partial n_{X^{\prime}}} d \mu\left(X^{\prime}, y^{\prime}\right)<+\infty,
$$

then the conclusions of Corollary 4.1 still hold true. 


\section{Applications to the representation theory}

As one application of Theorems 3.1 and 4.1, we obtain the representation theory of superharmonic functions in a cylinder. Let $u(V)$ be a function in $\mathbb{H}(\Gamma)$, where $V=(X, y)$, its Nevanlinna norm is defined by

$$
N_{u}(y):=\int_{\Gamma} u(V) \phi(X) d \Gamma
$$

The finite or infinite limits

$$
\lim _{y \rightarrow-\infty} e^{\sqrt{\lambda} y} N_{u}(y) \text { and } \lim _{y \rightarrow+\infty} e^{-\sqrt{\lambda} y} N_{u}(y)
$$

are denoted by $\mathscr{U}_{u}$ and $\mathscr{V}_{u}$, respectively.

We turn now to the proof of our main theorem.

Theorem 5.1. Let $u(V)$ be a nonnegative superharmonic function in $\mathbb{H}(\Gamma)$. Then we have for any $V=(X, y) \in \mathbb{H}(\Gamma)$

$$
u(V)=\mathscr{U}_{u} e^{-\sqrt{\lambda} y} \phi(X)+\mathscr{V}_{u} e^{\sqrt{\lambda} y} \phi(X)+\mathcal{G}_{\Gamma} \nu(V)+\mathcal{P} \mathcal{I}_{\Gamma} \mu(V)
$$

where $\nu$ is a unique positive measure in $\mathrm{H}(\Gamma)$ satisfying (3.17) and $\mu$ is a unique positive measure in $\partial \mathrm{H}(\Gamma)$ satisfying (4.10).

Proof. The proof is essentially contained in [9, Lemma 3]. Here we establish a much more direct approach to prove it, which is different from the previous one.

For harmonic functions $\mathscr{U}_{u} e^{-\sqrt{\lambda} y} \phi(X)$ and $\mathscr{V}_{u} e^{\sqrt{\lambda} y} \phi(X)$ in $\mathrm{H}(\Gamma)$, define

$$
w(V)=u(V)-\mathscr{U}_{u} e^{-\sqrt{\lambda} y} \phi(X)-\mathscr{V}_{u} e^{\sqrt{\lambda} y} \phi(X),
$$

where $V=(X, y) \in \mathbb{H}(\Gamma)$. It follows that $w(V)$ is a nonnegative superharmonic functions in $\mathbb{H}(\Gamma)$ and

$$
\mathscr{U}_{w}=\mathscr{V}_{w}=0
$$

By applying the Riesz decomposition theorem (see $[6])$ to $w(V)$ on $\mathbb{H}(\Gamma ;(-R, R)$ ), where $R>0$, we know that

$$
w(V)=w_{1}(V)+w_{2}(V)+w_{3}(V)+w_{4}(V)
$$

where

$$
\begin{aligned}
& w_{1}(V)=\int_{\partial \mathbb{H}(\Gamma ;(-R, R))} \frac{\partial \mathcal{G}_{\mathbb{H}(\Gamma,(-R, R))}(V, W)}{\partial n_{W}} d \mu_{0}(W), \\
& w_{2}(V)=\int_{\mathbb{H}(\Gamma ;(-R, R))} \mathcal{G}_{\mathbb{H}(\Gamma,(-R, R))}(V, W) d \nu_{0}(W), \\
& w_{3}(V)=-\int_{\Gamma} \frac{\partial \mathcal{G}_{\mathbb{H}(\Gamma,(-R, R))}\left(V,\left(X^{\prime}, R\right)\right)}{\partial y} d \Gamma
\end{aligned}
$$

and

$$
w_{4}(V)=\int_{\Gamma} \frac{\partial \mathcal{G}_{\mathrm{H}(\Gamma,(-R, R))}\left(V,\left(X^{\prime},-R\right)\right)}{\partial y} d \Gamma,
$$

where $d \nu_{0}$ is the Riesz measure and $d \mu_{0}$ is a positive measure on $\partial \mathbb{H}(\Gamma)$.

Case I. $w(V)<+\infty$. It is easy to see that $\mathcal{G}_{\mathbb{H}(\Gamma,(-R, R))}(V, W) \rightarrow \mathcal{G}_{\Gamma}(V, W)$ as $R \rightarrow+\infty$. So we have

$$
w_{1}(V) \rightarrow \mathcal{P} \mathcal{I}_{\Gamma} \mu_{0}(V)<+\infty
$$


and

$$
w_{2}(V) \rightarrow \mathcal{G}_{\Gamma} \nu_{0}(V)<+\infty
$$

as $R \rightarrow+\infty$.

It follows from (5.2) that there exist two sequences of points $V_{i}^{1}=\left(X_{i}^{1}, y_{i}^{1}\right) \in$ $\mathbb{H}(\Gamma ;(-R, R))\left(y_{i}^{1} \rightarrow+\infty\right)$ and $V_{i}^{2}=\left(X_{i}^{2}, y_{i}^{2}\right) \in \mathbb{H}(\Gamma ;(-R, R))\left(y_{i}^{2} \rightarrow-\infty\right)$ as $i \rightarrow+\infty$, such that

$$
w\left(V_{i}^{1}\right) \lesssim e^{\sqrt{\lambda} y_{i}^{1}} \phi\left(X_{i}^{1}\right) \epsilon_{i}
$$

and

$$
w\left(V_{i}^{2}\right) \lesssim e^{-\sqrt{\lambda} y_{i}^{2}} \phi\left(X_{i}^{2}\right) \epsilon_{i}
$$

respectively, where $\epsilon_{i} \rightarrow 0$ as $i \rightarrow+\infty$.

If we take $y_{i}^{1 *}=y_{i}^{1}+1$ and $y_{i}^{2 *}=y_{i}^{2}-1$, then we have

$$
w\left(V_{i}^{1}\right) \gtrsim-\int_{\Gamma} \frac{\partial \mathcal{G}_{\mathrm{H}\left(\Gamma,\left(-y_{i}^{1 *}, y_{i}^{1 *}\right)\right)}^{a}\left(V_{i}^{1},\left(X_{i}^{1 *}, y_{i}^{1 *}\right)\right)}{\partial y} d \Gamma \gtrsim \phi\left(X_{i}^{1}\right) \int_{\Gamma} \phi\left(X_{i}^{1 *}\right) d \Gamma
$$

and

$$
w\left(V_{i}^{2}\right) \gtrsim \int_{\Gamma} \frac{\partial \mathcal{G}_{\mathrm{H}\left(\Gamma,\left(-y_{i}^{2 *}, y_{i}^{2 *}\right)\right)}^{a}\left(V_{i}^{2},\left(X_{i}^{2 *}, y_{i}^{2 *}\right)\right)}{\partial y} d \Gamma \gtrsim \phi\left(X_{i}^{2}\right) \int_{\Gamma} \phi\left(X_{i}^{2 *}\right) d \Gamma
$$

from Lemma 4.3, which together with (5.3) and (5.4), yield that

$$
\int_{\Gamma} \phi\left(X_{i}^{1 *}\right) d \Gamma \lesssim e^{\sqrt{\lambda} y_{i}^{1}} \epsilon_{i}
$$

and

$$
\int_{\Gamma} \phi\left(X_{i}^{2 *}\right) d \Gamma \lesssim e^{-\sqrt{\lambda} y_{i}^{2}} \epsilon_{i}
$$

respectively.

Combining (5.5) and (5.6), we obtain

$$
w_{3}\left(V_{i}^{1}\right) \lesssim e^{\sqrt{\lambda} y_{i}^{1}} \phi\left(X_{i}^{1}\right) \epsilon_{i}
$$

and

$$
w_{4}\left(V_{i}^{2}\right) \lesssim e^{-\sqrt{\lambda} y_{i}^{2}} \phi\left(X_{i}^{2}\right) \epsilon_{i}
$$

from Lemma 4.3, respectively, which tend to 0 as $i \rightarrow+\infty$.

By letting $i \rightarrow+\infty$, we know that

$$
w(V)=\mathcal{P} \mathcal{I}_{\Gamma} \mu_{0}(V)+\mathcal{G}_{\Gamma} \nu_{0}(V)
$$

for all $V \in \mathbb{H}(\Gamma)$.

Case II. $w(V)=+\infty$. Note that the sum of $w_{1}(V)$ and $w_{2}(V)$ is infinite. We see that $w_{3}(V)$ and $w_{4}(V)$ are bounded from (5.7) and (5.8). This implies that $w(V)$ remains infinite as $R \rightarrow+\infty$, which shows that (5.9) holds.

It is evident that $d \mu_{0}$ and $d \nu_{0}$ are same for the functions $w(V)$ and $u(V)$, respectively. Thus they are simply denoted by $d \mu$ and $d \nu$, respectively. Finally, we complete the proof of Theorem 5.1.

Theorems 3.1 and 4.1 immediately give the following result. 
Corollary 5.1. Let $u(V)(\not \equiv+\infty)$ be defined by (5.1) and $\gamma>n-2$, where $V=(X, y) \in \mathbb{H}(\Gamma)$. Then the subset $\mathcal{E}\left(\epsilon ; \sigma_{1}(\mu, \nu), \gamma\right)\left(\right.$ resp. $\left.\mathcal{E}\left(\epsilon ; \sigma_{2}(\mu, \nu), \gamma\right)\right)$ of $\mathbb{H}(\Gamma)$ has a covering $\left\{r_{j}\right\}_{j=0}^{\infty}$ satisfying (3.8) such that

$$
\begin{gathered}
\lim _{\substack{y \rightarrow-\infty, V=(X, y) \in \mathrm{H}(\Gamma)-\mathcal{E}\left(\epsilon ; \sigma_{1}(\mu, \nu), \gamma\right)}} e^{\sqrt{\lambda} y} \phi^{n-\gamma-1}(X)\left\{u(V)-\mathscr{U}_{u} e^{-\sqrt{\lambda} y} \phi(X)-\mathscr{V}_{u} e^{\sqrt{\lambda} y} \phi(X)\right\}=0 . \\
\left(\text { resp. } \lim _{\substack{y \rightarrow+\infty, V=(X, y) \in \mathbb{H}(\Gamma) \\
-\mathcal{E}\left(\epsilon ; \sigma_{2}(\mu, \nu), \gamma\right)}} e^{-\sqrt{\lambda} y} \phi^{n-\gamma-1}(X)\left\{u(V)-\mathscr{U}_{u} e^{-\sqrt{\lambda} y} \phi(X)-\mathscr{V}_{u} e^{\sqrt{\lambda} y} \phi(X)\right\}=0 .\right)
\end{gathered}
$$

\section{Applications to the geometrical property of rarefied set}

As another application of Theorems 3.1 and 4.1, the present section is to devoted to the study of the geometrical property of rarefied set at $+\infty$. To this end we first recall the following definition.

It is known that the Martin boundary of $\mathbb{H}(\Gamma)$ is the set $\partial \mathbb{H}(\Gamma) \cup\{ \pm \infty\}$, and the Martin function at $+\infty$ with respect to a reference point chosen suitably is given by (see $[10])$

$$
\mathcal{M}_{\Gamma}(V,+\infty)=e^{\sqrt{\lambda} y} \phi(X),
$$

where $V=(X, y) \in \mathbb{H}(\Gamma)$.

Definition 6.1. (see [9]) If there exists a positive superharmonic function $\nu(V)$ in $\mathbb{H}(\Gamma)$ such that

$$
\inf _{V \in \mathbb{H}(\Gamma)} \frac{v(V)}{\mathcal{M}_{\Gamma}(V,+\infty)}=0
$$

and

$$
E \subset\left\{V=(X, y) \in \mathbb{H}(\Gamma) ; v(V) \geq e^{\sqrt{\lambda} y}\right\},
$$

then a subset $E$ of $\mathbb{H}(\Gamma)$ is said to be rarefied at $+\infty$ in $\mathbb{H}(\Gamma)$.

With this definition in mind, we state the main theorem of the present section. We refer the readers to [10, Theorem 3.1] for the geometrical properties of minimally thin set at $+\infty$.

Theorem 6.1. Let a subset $E$ be rarefied at $+\infty$ in $\mathrm{H}(\Gamma)$. Then it has a covering $\left\{r_{j}\right\}_{j=0}^{\infty}$ satisfying

$$
\sum_{j=0}^{\infty}\left(r_{j}\right)^{n-1}<+\infty .
$$

Proof. Definition 6.1 gives that there exists a positive superharmonic function $v(V)$ in $\mathrm{H}(\Gamma)$ such that (6.1) and (6.2) hold. And then it is represented as

$$
v(V)=\mathscr{U}_{u} e^{-\sqrt{\lambda} y} \phi(X)+\mathcal{G}_{\Gamma} \nu^{\prime}(V)+\mathcal{P I}_{\Gamma} \mu^{\prime}(V)
$$

from Theorem 5.1 and (6.1), where $\nu^{\prime}$ is a unique positive measure in $\mathbb{H}(\Gamma)$ and $\mu^{\prime}$ is a unique positive measure in $\partial \mathbb{H}(\Gamma)$.

Three sets

$\left\{V=(X, y) \in \mathbb{H}(\Gamma) ; 3 \mathscr{U}_{u} e^{-2 \sqrt{\lambda} y} \phi(X) \geq 1\right\}, \quad\left\{V=(X, y) \in \mathbb{H}(\Gamma) ; 3 \mathcal{G}_{\Gamma} \nu^{\prime}(V) \geq e^{\sqrt{\lambda} y}\right\}$ and

$$
\left\{V=(X, y) \in \mathbb{H}(\Gamma) ; 3 \mathcal{P} \mathcal{I}_{\Gamma} \mu^{\prime}(V) \geq e^{\sqrt{\lambda} y}\right\}
$$

are denoted by $E^{(1)}, E^{(2)}$ and $E^{(3)}$, respectively. It follows that

$$
E \subset E^{(1)} \cup E^{(2)} \cup E^{(3)}
$$


from (6.2).

For each $E^{(i)}(i=1,2,3)$, it is evident that there shall exist a covering which covers it. It follows that the set $E^{(1)}$ has a covering $\left\{r_{1}^{(1)}\right\}$ satisfying

$$
r_{1}^{(1)}<+\infty
$$

from the boundness of $E^{(1)}$.

We apply Theorems 3.1 and 4.1 with two measures $\nu$ and $\mu$, where $\nu=3 \nu^{\prime}$ and $\mu=3 \mu^{\prime}$, respectively. And then there exist two positive constants $L$ and $\epsilon$ satisfying

$$
E^{(2)} \cap \mathbb{H}(\Gamma ;(L,+\infty)) \subset \mathcal{E}\left(\epsilon ; \sigma_{2}(\nu), n-1\right)
$$

and

$$
E^{(3)} \cap \mathbb{H}(\Gamma ;(L,+\infty)) \subset \mathcal{E}\left(\epsilon ; \sigma_{2}(\mu), n-1\right),
$$

respectively.

It follows from Lemma 3.3 that two subsets $\mathcal{E}\left(\epsilon ; \sigma_{2}(\nu), n-1\right)$ and $\mathcal{E}\left(\epsilon ; \sigma_{2}(\mu), n-1\right)$ have two coverings $\left\{r_{j}^{(2)}\right\}_{j=1}^{\infty}$ and $\left\{r_{j}^{(3)}\right\}_{j=1}^{\infty}$, respectively, which satisfy

$$
\sum_{j=1}^{\infty}\left(r_{j}^{(2)}\right)^{n-1}<+\infty \quad \text { and } \quad \sum_{j=1}^{\infty}\left(r_{j}^{(3)}\right)^{n-1}<+\infty
$$

respectively.

Hence $E^{(2)}$ and $E^{(3)}$ have also two coverings $\left\{r_{j}^{(2)}\right\}_{j=0}^{\infty}$ and $\left\{r_{j}^{(3)}\right\}_{j=1}^{\infty}$ with an additional covering $r_{0}^{(2)}$ covering $\mathbb{H}(\Gamma ;[0, L))$, respectively, which satisfy

$$
\sum_{j=0}^{\infty}\left(r_{j}^{(2)}\right)^{n-1}<+\infty \quad \text { and } \quad \sum_{j=1}^{\infty}\left(r_{j}^{(3)}\right)^{n-1}<+\infty
$$

respectively.

By rearranging three coverings $\left\{r_{1}^{(1)}\right\},\left\{r_{j}^{(2)}\right\}_{j=0}^{\infty}$ and $\left\{r_{j}^{(3)}\right\}_{j=1}^{\infty}$, the set $E$ has a covering $\left\{r_{j}\right\}_{j=0}^{\infty}$ from (6.4), which satisfies (6.3) from (6.5) and (6.6).

\section{Example}

As mentioned above, usually it is very hard to obtain geometrical properties of the rarefied set at $+\infty$. The new results proved in this paper provide us an efficient way to obtain the covering theorem. We end the paper with following example, which will show that the reverse of Theorem 6.1 is not true.

Example 7.1. Let

$$
r_{j}=j^{\frac{1}{2-n}} \quad \text { and } \quad y_{j}=j+1,
$$

where $j=1,2,3, \ldots$. And the covering $\left\{r_{j}\right\}_{j=1}^{\infty}$ satisfies

$$
\sum_{j=1}^{\infty}\left(r_{j}\right)^{n-1}=\sum_{j=1}^{\infty} j^{-\frac{n-1}{n-2}}<+\infty .
$$

Put $\overline{\Gamma^{\prime}} \subset \Gamma$. Suppose that

$$
B_{j} \subset \mathbb{H}\left(\Gamma^{\prime}\right) \quad \text { and } \quad y_{j} \geq 2 r_{j}
$$

for every $j \geq j_{0}$, where $j_{0}$ is an integer. 
If we define

$$
E:=\bigcup_{j=j_{0}}^{\infty} B_{j}
$$

then it is not rarefied at $+\infty$ in $\mathbb{H}(\Gamma)$.

Proof. There exist a positive integer $j_{0}$ such that $\mathcal{M}_{\Gamma}(V,+\infty) \gtrsim e^{\sqrt{\lambda} y_{j}}$ for any $j \geq j_{0}$, where $y_{j} \geq 2 r_{j}$. And hence we have for any $V \in \bar{B}_{j}$

$$
\hat{R}_{\mathcal{M}_{\Gamma}(\cdot, \infty)}^{B_{j}}(V) \gtrsim e^{\sqrt{\lambda} y_{j}}
$$

where $j \geq j_{0}$.

There exists a measure $\tau$ on $C_{n}(\Gamma)$ such that supp $\tau \subset \bar{B}_{j}$ and $\tau\left(\bar{B}_{j}\right)=1$. So we have for any $W \in \bar{B}_{j}$

$$
\int_{\mathbb{H}(\Gamma)}|V-W|^{2-n} d \tau(V)=\left\{\operatorname{Cap}\left(\bar{B}_{j}\right)\right\}^{-1} .
$$

Combining (3.15) (7.1) and (7.2), we have

$$
\begin{aligned}
\left\{\operatorname{Cap}\left(\bar{B}_{j}\right)\right\}^{-1} \lambda_{B_{j}}(\mathbb{H}(\Gamma)) & =\int\left(\int|V-W|^{2-n} d \tau(V)\right) d \lambda_{B_{j}}(W) \\
& \gtrsim \int\left(\int \mathcal{G}_{\Gamma}(V, W) d \lambda_{B_{j}}(W)\right) d \tau(V) \\
& =\int \hat{R}_{\mathcal{M}_{\Gamma}(\cdot, \infty)}^{B_{j}} d \tau(V) \gtrsim e^{\sqrt{\lambda} y_{j}}
\end{aligned}
$$

which gives that

$$
\lambda_{B_{j}}(\mathbb{H}(\Gamma)) \gtrsim \operatorname{Cap}\left(\bar{B}_{j}\right) e^{\sqrt{\lambda} y_{j}} \gtrsim r_{j}^{n-2} e^{\sqrt{\lambda} y_{j}},
$$

because $\operatorname{Cap}\left(\bar{B}_{j}\right)=r_{j}^{n-2}$.

Since $\lambda_{\mathcal{E}_{j}}(\mathbb{H}(\Gamma))=\lambda_{B_{j}}(\mathbb{H}(\Gamma))$, we have

$$
\sum_{j=j_{0}}^{\infty} e^{-\sqrt{\lambda} y_{j}} \lambda_{\mathcal{E}_{j}}(\mathbb{H}(\Gamma)) \gtrsim \sum_{j=j_{0}}^{\infty} e^{\sqrt{\lambda}\left(y_{j}-j\right)} r_{j}^{n-2}=\sum_{j=j_{0}}^{\infty} j^{-1}=+\infty,
$$

which together with Theorem 1 in [9], gives that $E$ is not rarefied at $+\infty$ in $\mathbb{H}(\Gamma)$.

Example 7 and Theorem 6.1 immediately give the following result.

Corollary 7.1. Let $u(V)$ be as in Theorem 5.1. Then $u(V) e^{\sqrt{\lambda} y}$ uniformly converges to $c_{+\infty}(u) \phi(X)$ as $y \rightarrow+\infty$ outside a set which has a covering $\left\{r_{j}\right\}_{j=0}^{\infty}$ satisfying (6.3), where

$$
c_{+\infty}(u)=\inf _{V \in \mathbb{H}(\Gamma)} \frac{u(V)}{\mathcal{M}_{\Gamma}(V,+\infty)} .
$$

Acknowledgments. The author would like to thank Associate Editor and the referee for their time and effort spent on this paper. The author also thanks the referee for his precious and thoughtful suggestions on this paper, which has largely improved the presentation of the paper. 


\section{References}

[1] Azarin, V.S.: Generalization of a theorem of Hayman's on a subharmonic function in an $n$-dimensional cone. - Mat. Sb. (N.S.) 108:66, 1965, 248-264 (in Russian).

[2] Courant, R., and D. Hilbert: Methods of mathematical physics. Vol. I. - Interscience Publishers, Inc., New York, N.Y., 1953.

[3] Gergün, S., and I. V. OstrovskiI: On the Poisson representation of a function harmonic in the upper half-plane. - Comput. Methods Funct. Theory 2:1, 2002, 191-213.

[4] Gergün, S., and I. V. Ostrovskit: On the Poisson representation of a function harmonic in the upper half-plane. - Mat. Fiz. Anal. Geom. 9:2, 2002, 243-248.

[5] Gilbarg, D., and N.S. Trudinger: Elliptic partial differential equations of second order. Grundlehren Math. Wiss. 224, Springer-Verlag, Berlin-New York, 1977.

[6] Helms, L. L.: Introduction to potential theory. - Pure and Applied Mathematics, Vol. XXII, Wiley-Interscience A Division of John Wiley \& Sons, New York-London-Sydney, 1969.

[7] Huang, J.: Growth property at infinity of the maximum modulus with respect to the Schrödinger operator. - Internat. J. Math. 27:2, 2016, 1650009, 1-11.

[8] LiN, L., and J. Lu: Decay estimates of discretized Green's functions for Schrödinger type operators. - Sci. China Math. 59:8, 2016, 1561-1578.

[9] Мiяamoto, I.: Two criteria of Wiener type for minimally thin sets and rarefied sets in a cylinder. - Hokkaido Math. J. 37:3, 2007, 507-534.

[10] Miyamoto, I., and M. Yanagishita: Some characterizations of minimally thin sets in a cylinder and Beurling-Dahlberg-Sjögren type theorems. - Proc. Amer. Math. Soc. 133:5, 2005, 1391-1400.

[11] Mizuta, Y.: Potential theory in Euclidean spaces. - GAKUTO International Series, Mathematical Sciences and Applications 6, Gakkōtosho Co., Ltd., Tokyo, 1996.

[12] Qiao, L.: Matsaev's type theorems for solutions of the stationary Schrödinger equation and its applications. - Discrete Contin. Dyn. Syst. 36:10, 2016, 5709-5720.

[13] Qiao, L.: Asymptotic behaviors of Green-Sch potentials at infinity and its applications. Discrete Contin. Dyn. Syst. Ser. B 22:6, 2017, 2321-2338.

[14] Qiao, L.: Asymptotic behavior of Poisson integrals in a cylinder and its application to the representation of harmonic functions. - Bull. Sci. Math. 144, 2018, 39-54.

[15] QiaO, L., and G. PAN: Lower-bound estimates for a class of harmonic functions and applications to Matsaev's type theorem. - Bull. Sci. Math. 140:4, 2016, 70-85.

[16] Ramachandran, K.: Asymptotic behavior of positive harmonic functions in certain unbounded domains. - Potential Anal. 41:2, 2014, 383-405.

[17] Rozenblum, G. V., M. A. Shubin, and M. Z. Solomyak: Partial differential equations. VII: Spectral theory of differential operators. - Encyclopaedia Math. Sci. 64, Springer-Verlag, 1994.

[18] WANG, K.: On the Neumann problem for harmonic functions in the upper half plane. - J. Math. Anal. Appl. 419:2, 2014, 839-848.

[19] Yang, D., and Y. Ren: Dirichlet problem on the upper half space. - Proc. Indian Acad. Sci. (Math. Sci.) 124:2, 2014, 175-178.

[20] Yoshida, H.: Nevanlinna norm of a subharmonic function on a cone or on a cylinder. - Proc. London Math. Soc. (3) 54:2, 1987, 267-299.

[21] Yoshida, H.: Harmonic majorization of a subharmonic function on a cone or on a cylinder. Pacific J. Math. 148:2, 1991, 369-395. 